\title{
Bilateral Posterior and Lateral Semicircular Canals Aplasia Associated with Other Inner Ear Malformation in A 2-Year-Old Child: A Rare Case Report
}

\author{
Fatimah Albazroun*, Zahrah Almoumen and Hazim Al Eid \\ Department of ENT, Dammam, Saudi Arabia \\ *Corresponding author: Fatimah Albazroun, Department of ENT, Dammam, Saudi Arabia
}

Received: 笽December 13, 2018

Published: 海 December 19, 2018

\begin{abstract}
Inner ear malformation accounts for approximately $20 \%$ of reported cases of congenital sensorineural deafness. It results from developmental arrest in different stages of embryogenesis. Aplasia of one of the semicircular canals with or without cochlear malformation is well known and has been reported in journals. However; to our knowledge, aplasia of two semicircular canals have been rarely reported, and for this reason the clinical reflection of this anomaly is poorly understood. This case will be hopefully used to gain an in-depth understanding of illness and help in the diagnosis and proper management of future cases. In this paper we present a report concerns a 2-year-old boy with no family history of hearing loss, presenting with bilateral profound sensorineural hearing loss with no other congenital anomalies. CT scan and MRI shows dysplastic bilateral vestibules, bilateral hypoplastic cochlea and bilateral lateral and posterior semicircular canal (SCC) aplasia.
\end{abstract}

Keywords: Semicircular canal aplasia; Sensorineural hearing loss; Ct Scan, Cochlear hypoplasia

Abbreviations: SNHL: Sensorineural Hearing Loss; LSCS: Lower Segment Caesarean Section; ECV: Ear Canal Volume; Ct: Computerized Tomography; CVEMP: Completed Vestibular Evoked Myogenic Potential; MRI: Magnetic Resonance Imaging

\section{Introduction}

Inner ear in embryo starts to develop during $3^{\text {rd }}$ week of gestation. A structure called 'otic placodes' which arise from the surface ectoderm on each side of the rhombencephalon represent the fundamental embryological part of inner air developmental. The otic placodes subsequently invaginate and form otocysts. Diverticulum buds from the otocysts form the endolymphatic sacs, which eventually by the $5^{\text {th }}$ week; form the cochlea and vestibules, while utricle segments of the otocysts form the semicircular canals by the end of the $7^{\text {th }}$ week. The Superior semicircular canal develops first while the lateral develops last [1]. Premature arrest in different parts of development leads to inner ear malformation. The earlier the arrest the more sever the deformity [2]. Morphologically inner ear malformation is classified into two categories; membranous malformations which involve the inner ear hair cells and therefore can't be diagnosed based on CT or MRI imaging of the temporal bone. And bony labyrinth malformation which can be radiologically demonstrated [3].

\section{Case Report}

We report the case of one of the non-identical twin brothers: a 2-year-old boy, born of a consanguineous marriage to medically free parents, was first seen and diagnosed in our institution when he was 1-year-old to have bilateral profound SNHL. His hearing loss was first noticed by his mother when he was five months of age. She described him to be a 'Hard of hearing' that even loud sounds fail to startle him compared to his twin brother. Since the diagnosis, the patient has been in regular follow-up at our ENT outpatient department. His parents had not reported any active complains about him up until he started walking, they stated that he had unsteady gait and poor balance which usually followed by Falls or in some occasions 'a fainting episode' which they have never noticed with his twin brother. Other than speech delay; the patient had displayed normal developmental milestones appropriate to his age. He was born at term by LSCS with birth weight of $2.4 \mathrm{~kg}$. Apart from gestational induced hypertension, his mother had uneventful 
pregnancy. His parents reported no family history of hearing loss, he has 7 healthy siblings. Examination of the face showed frontal bossing and depressed nasal bridge. Right ear, nose and throat examination were normal, while left ear examination revealed otitis media with effusion. Tympanogram was performed; it revealed type B with normal ECV bilaterally. ABR was done, it showed bilateral unidentifiable repeatable (I, iii, v) waveforms at $90 \mathrm{dBHI}$ and low wave V was traced down to $20 \mathrm{dBHI}$. Radiological examination CT scan of temporal bone showed bilateral aplasia of the posterior semicircular canal and lateral semicircular canals, dysplastic bilateral vestibules (Figure 1) and bilateral cystic fusion of the middle and apical turns of the cochlea (Figure 2). MRI confirmed
CT scan finding, and additionally showed normal cochlear nerve bilaterally. In addition to that, patient CVEMP in a seated position in respond to tone burst stimuli $800 \mathrm{~Hz}$ frequency with a two-cycle rise/fall and no plateau (black mam gated) it exhibited lowered threshold on left side compared to right side $(80 \mathrm{dBnHL}$ versus $95 \mathrm{dBnHL}$ ) with no significant intraoral asymmetry ration $25 \%$ to the left. Molecular genetic analysis of the patient detected the heterozygous variants c.362T $>\mathrm{A}$ p(Val121Glu) and c.3467G $>\mathrm{A}$ $\mathrm{p}$ (Cys1156Tyr) in the OTOG and OTOGL genes, respectively. Additionally, potentially relevant variants were detected; they are reported in the table below (Table 1 ).
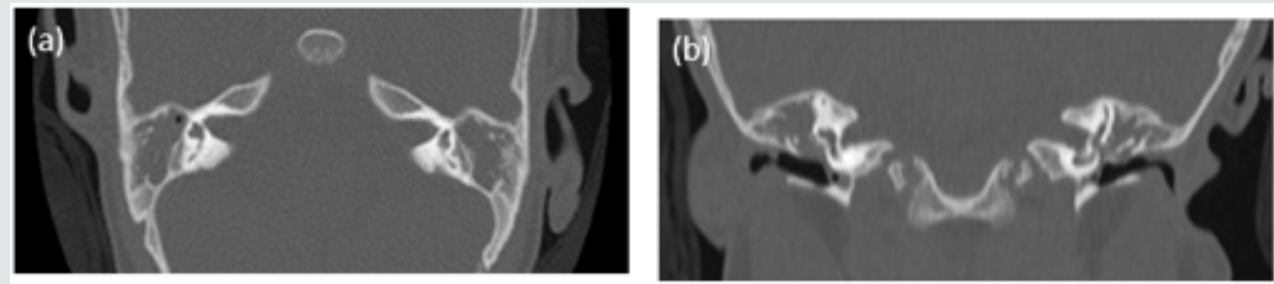

Figure 1: High resolution computed tomography (a) axial view, showing aplasia of the lateral semicircular canal and dysplastic vestibule bilaterally. (b) coronal view.
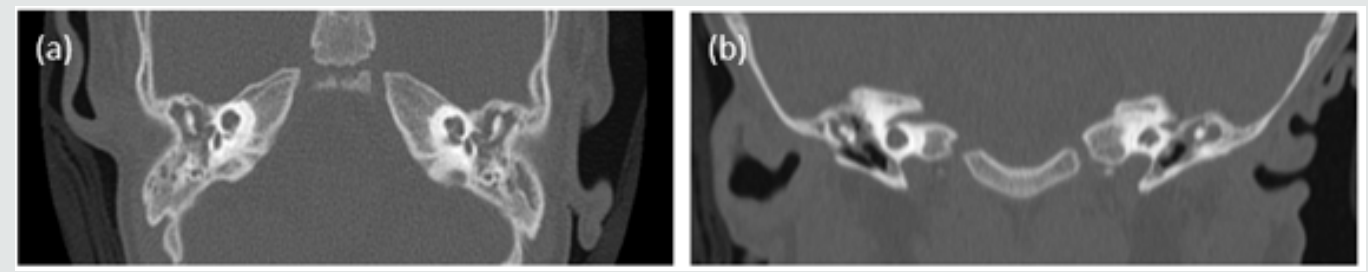

Figure 2: High resolution computed tomography (a) axial view, cystic fusion of the middle and apical turns of the cochlea bilaterally. (b) coronal view.

Table 1: Potentially Relevant Variants.

\begin{tabular}{|c|c|c|c|c|c|}
\hline Variant (HGVS) & Protein (HGVS) & Zyg & Reported (literature/DB) & GnomAD & Classification \\
\hline NM_004525.2(LRP2): c.1973A>G & p. (Tyr658Cys) & Het. & ClinVar:332195 & $0.089 \%$ & VOUS \\
\hline NM_001145026.1(PTPRQ): c.6568G>T & p. (Ala2190Ser) & Het. & DbNSP: rs199746225 & $0.035 \% 64$ het.; 2hom & VOUS \\
\hline NM_0033056.3(PCDH15): c.2990A>G & p. (Glu997Gly) & Het. & ClinVar: 46456 & $0.032 \%$ & VOUS \\
\hline NM_000091.4(COL4A3): c.1863A>G & p. (Gln612=) & Het. & DbNSP: rs771390525 & $0.022 \%$ & VOUS \\
\hline
\end{tabular}

\section{Discussion}

Table 2: Classification of inner ear malformations according to Jackler.

\begin{tabular}{|l|r|}
\hline Category A & Aplasia or Malformation of Cochlea \\
\hline & Aplasia of cochlea, normal or deformed vestibule and semicircular system. \\
\hline & Hypoplasia of cochlea, normal or deformed vestibule and semicircular system \\
\hline & Incomplete cochlea, normal or deformed vestibule and semicircular system \\
\hline Category B & Nomon cavity: choclea and vestibule build a common space without internal architecture, normal or deformed semicircular system \\
\hline & Dysplasia of vestibule and lateral semicircular canal, normal anterior and posterior semicircular canal. \\
\hline & Enlarged vestibular aqueduct and normal or dilated vestibule, normal semicircular system. \\
\hline
\end{tabular}


Inner ear malformations accounts for $20 \%$ of cases that present with congenital hearing loss [4]. Jacker et al. classified bony labyrinths malformation in 1987 [3], where he grouped inner ear malformation into two categories; category A which contains malformations associated with aplasia of the cochlea. category B; which contains malformation associated with normal cochlea [5]. The classification is illustrated in the table (Table 2). Cochlear Hypoplasia which accounts for $15 \%$ of cochlear malformations define as; 'cochlea with 1.5 turns' where the dimensions of the cochlea are less than those of a normal cochlea with other various architecture deformities [5]. There are Four types of cochlear hypoplasia:

a) $\mathrm{CH}-\mathrm{I}$ (Bud-like cochlea)

b) CH-II (Cystic hypoplastic cochlea)

c) $\mathrm{CH}-\mathrm{III}$ (Cochlea with less than 2 turns)

d) CH-IV (Cochlea with hypoplastic middle and apical turns)

Most patients with cochlear hypoplasia have severe to profound hearing loss, which make them candidates for cochlear implant if cochlear nerve was normal. In case of bilateral hypoplasia, it's important to know which ear has less malformation and larger cochlear nerve. Intraoperatively, facial nerve malposition is anticipated finding as hypoplasia of the cochlea are usually associated with semicircular abnormalities. Surgeons are usually using thin and shorter electrodes to insert in the cochlea as the numbers of turns are smaller and narrower. Unfortunately, Patients with cochlear hypoplasia are at higher intraoperative risks, such as cerebrospinal fluid leakage, meningitis, and electrode displacement [3]. Other inner ear abnormalities such as semicircular canals aplasia is not that common compared to dysplasia. It's usually associated with abnormal course of the facial nerve, atresia of the oval window, and abnormal ossicles [6]. Aplasia of all semicircular canals can be seen in patients with CHARGE syndrome [5-7] while isolated aplasia of the posterior semicircular canal is seen in patients with Waardenburg syndrome and Alagille syndrome [5]. CT scan is the modality of choice to diagnose semicircular canal aplasia [5].

\section{Conclusion}

In conclusion, this is a case of a 2 -year-old patient with bilateral posterior and lateral semicircular canals aplasia with cochlear hypoplasia and vestibular dysplasia, apart from the inner ear malformation, no other congenital anomalies, patient underwent uneventful cochlear implantation surgery. From our side, we recommend that more patients with similar finding should be examined and investigated by genetic analysis for better delineation of this disorder and understanding of the cause [8].

\section{References}

1. Yiin RSZ, Tang PH, Tan TY (2011) Review of congenital inner ear abnormalities on CT temporal bone. The British Journal of Radiology 84(1005): 859-863.

2. Brookhouser PE (1993) Sensorineural hearing loss in children. In: Cummings CW, Harker LA, Chung Hee Shin (Eds.), Otolaryngology Head and Neck CT and MR Imagings of Semicircular Canal Aplasia -14 -Surgery, (2 $2^{\text {nd }}$ edn), pp. 3080-3102.

3. Levent Sennaroğlu, Münir Demir Bajin (2017) Classification and Current Management of Inner Ear Malformations. Balkan Med J 34(5): 397-411.

4. Sennaroglu L (2010) Cochlear implantation in inner ear malformations-a review article. Cochlear Implants Int 11(1): 4-41.

5. Shin CH, Hong HS, Yi BH, JG Cha, SJ Park, DH Kim, et al. (2009) CT and MR imagings of semicircular canal aplasia. J Korean Soc Radiol 61: 9-15.

6. Morimoto AK, Wiggins RH, Hudgins PA, Hedlund GL, Hamilton B, et al. (2006) Absent semicircular canals in CHARGE syndrome: radiologic spectrum of findings. AJNR Am J Neuroradiol 27(8): 1663-1671.

7. Bartel Friedrich S, Wulke C ((2008)) Classification and diagnosis of ear malformations. GMS Curr Top Otorhinolaryngol Head Neck Surg 6: 5.

8. Jackler RK, Luxford WM, House WF (1987) Congenital malformations of the inner ear: a classification based on embryogenesis. Laryngoscope 97(3 Pt 2): 2-14.

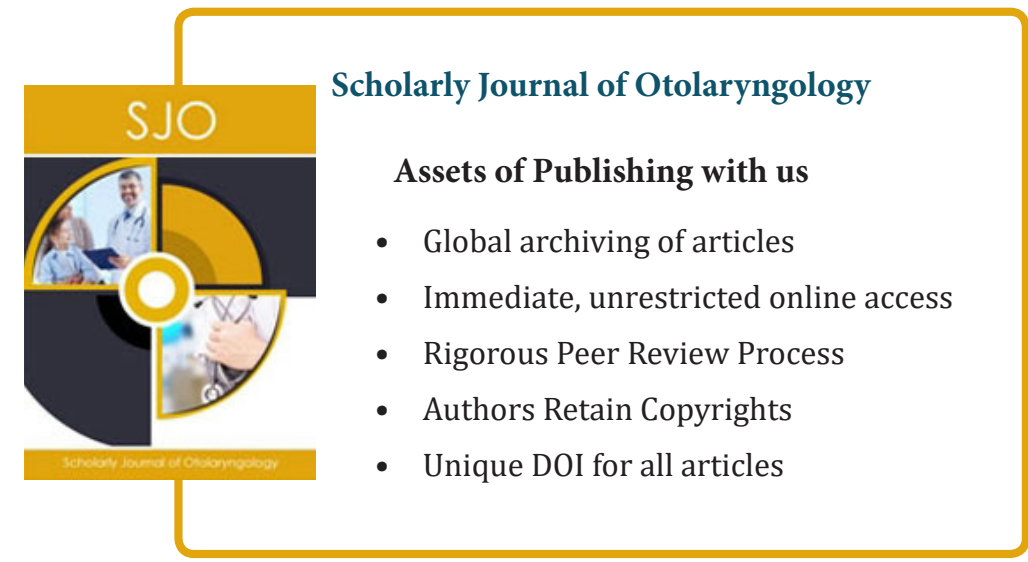

